\title{
Spirometra decipiens (Cestoda: Diphyllobothriidae) Collected in A Heavily Infected Stray Cat from the Republic of Korea
}

\author{
Hyeong-Kyu Jeon ${ }^{\dagger}$, Hansol Park’, Dongmin Lee, Seongjun Choe, Keeseon S. Eom* \\ Department of Parasitology, Medical Research Institute and Parasite Resource Bank, Chungbuk National University School of Medicine, \\ Cheongju 28644, Korea
}

\begin{abstract}
Morphological and molecular characteristics of spirometrid tapeworms, Spirometra decipiens, were studied, which were recovered from a heavily infected stray cat road-killed in Eumseong-gun, Chungcheongbuk-do (Province), the Republic of Korea (=Korea). A total of 134 scolices and many broken immature and mature proglottids of Spirometra tapeworms were collected from the small intestine of the cat. Morphological observations were based on 116 specimens. The scolex was 22.8-32.6 mm (27.4 mm in average) in length and small spoon-shape with 2 distinct bothria. The uterus was coiled 3-4 times, the end of the uterus was ball-shaped, and the vaginal aperture shaped as a crescent moon was closer to the cirrus aperture than to the uterine aperture. PCR amplification and direct sequencing of the cox 1 target fragment (377 bp in length and corresponding to positions 769-1,146 bp of the cox1 gene) were performed using total genomic DNA extracted from 134 specimens. The cox1 sequences (377 bp) of the specimens showed 99.0\% similarity to the reference sequence of $S$. decipiens and $89.3 \%$ similarity to the reference sequence of S. erinaceieuropaei. In the present study, we report a stray cat heavily infected with $S$. decipiens identified by mitochondrial cox 1 sequence analysis and morphological examinations of the adult worms.
\end{abstract}

Key words: Spirometra decipiens, scolex, molecular detection, cox1, cat

The stray or feral cat is a powerful predator in the ecosystem of wild life in Korea and has a wide range of food stuffs originating from mammalian, avian, reptilian, and amphibian animals. The free-roaming stray cats act as the potential source of environmental contamination and transmit the pathogenic parasites to humans even to domesticated cats as the reservoir host of zoonotic helminths. Among intestinal helminths, Spirometra species of cats are the most important in terms of public health. Spirometra species in cats have been reported sporadically by many authors in the Republic of Korea (= Korea). Kang (1967) examined 41 cats in Gyeongsangnam-do (Province) with helminth infections such as Clonorchis sinensis, Paragonimus sp. Taenia taeniaeformis, Spirometra sp., and Toxocara cati [1]. Huh et al. (1993) reported 7 helminth species, T. cati, Anisakis simplex larvae, C. sinensis, Pharyngostomum cordatum,

- Received 15 August 2017, revised 25 January 2018, accepted 2 February 2018.

*Corresponding author (kseom@chungbuk.ac.kr)

'These authors contributed equally to this work.

(c) 2018, Korean Society for Parasitology and Tropical Medicine

This is an Open Access article distributed under the terms of the Creative Commons Attribution Non-Commercial License (http://creativecommons.org/licenses/by-nc/4.0) which permits unrestricted non-commercial use, distribution, and reproduction in any medium, provided the original work is properly cited.
Spirometra erinaceieuropaei (formerly S. erinacei), and T. taeniaeformis, from 41 cats in Seoul [2]. Yang et al. (1995) detected 4 helminth species, T. cati, Diphyllobothrium latum, S. erinacei, and T. taeniaeformis, from 133 cats in Jeollanam-do (Province) [3]. Sohn and Chai [4] reported more than 29 helminth species from feral cats purchased from a market in Busan. In addition, Chai et al. [5] reported 4 nematode species, 23 trematode species, and 5 cestode species in cats. Thus, intestinal parasite infections in stray or feral cats have been reported involving more than 29 species of helminths, including 23 trematodes, 5 cestodes and 4 nematodes in Korea [4,5].

With respect to cestodes of cats, a total of 4 species, comprising S. erinaceieuropaei, a diphyllobothriid tapeworm, T. taeniaeformis, and Hymenolepis diminuta, have been commonly found in Korea [1-5]. S. erinaceieuropaei has been recognized as the only Spirometra species in Korea before the report of Jeon et al. [6]. Spirometra species inducing human sparganosis are being identified by morphological and genetic analyses, and the complete mitochondrial genomes of $S$. erinaceieuropaei and $S$. decipiens have been characterized and recorded in Korea [6,7]. The genetic distance of $S$. decipiens vs $S$. erinaceieuropaei and $S$. 
decipiens vs $S$. proliferum was estimated on the basis of partial cox1 sequences, respectively [8]. Presently, 2 Spirometra species were reported in Korea, therefore, identification of Spirometra species is required. In this study, we identified a Spirometra species from a stray cat by molecular analysis using cytochrome $c$ oxidase (cox1) in addition to morphological observations of mature proglottids.

A road-killed stray cat (Felis catus) was found in the Eumseong-gun area in Chungcheongbuk-do in November 2011. The small intestine was isolated and longitudinally opened with a pair of scissors in $0.85 \%$ saline and rinsed in PBS until the supernatant remained clear. The sediment containing the intestinal content was carefully examined under a stereomicroscope. Two types of worms were detected in the small intestines during the postmortem examination. The collected worms were fixed with $10 \%$ neutral buffered formalin under a glass slide with pressure for microscopic observations after carmine staining. Immature and mature proglottids of the tapeworms were pressed and fixed in alcohol-formalin-acetic acid (AFA) for carmine staining. Mature proglottids were observed with respect to the vaginal opening, uterus, uterine pore, cirrus, genital pore, testes, and vitellaria, based on the morpho- logical data of Faust et al. [9]. The scolices and proglottids were preserved at $-70^{\circ} \mathrm{C}$ until use for molecular identification.

A single scolex was chopped into small pieces, and total genomic DNA was extracted using a DNeasy tissue kit (Qiagen, Valencia, California, USA). PCR and DNA sequencing for differential diagnosis were performed according to established protocols $[9,10]$. Pairwise genetic distances on the partial mitochondrial cox1 sequences ( $377 \mathrm{bp}$ ) were analyzed using the neighbor-joining method with Kimura's 2-substitution model. The partial mitochondrial cox1 sequences ( $377 \mathrm{bp)}$ of 4 taxa of Diphyllobothriidae comprised S. decipiens (GenBank no. KJ599679), S. erinaceieuropaei (GenBank no. KJ599680), Diphyllobothrium nihonkaiense (GenBank no. EF420138), and Diphyllobothrium latum (GenBank no. DQ985706). The parasite materials (PRB000720) used in the present study were provided by the Parasite Resource Bank of Korea, National Research Resource Center, Korea.

A total of 134 scolices and mature proglottids of Spirometra species were found in the cat examined. Morphological observations were based on 116 scolices and mature proglottids. The scolex was $2.74 \pm 0.43 \mathrm{~mm} \times 0.73 \pm 0.11 \mathrm{~mm}$ in average size and small spoon-shape with 2 distinct bothria (Fig. 1A).

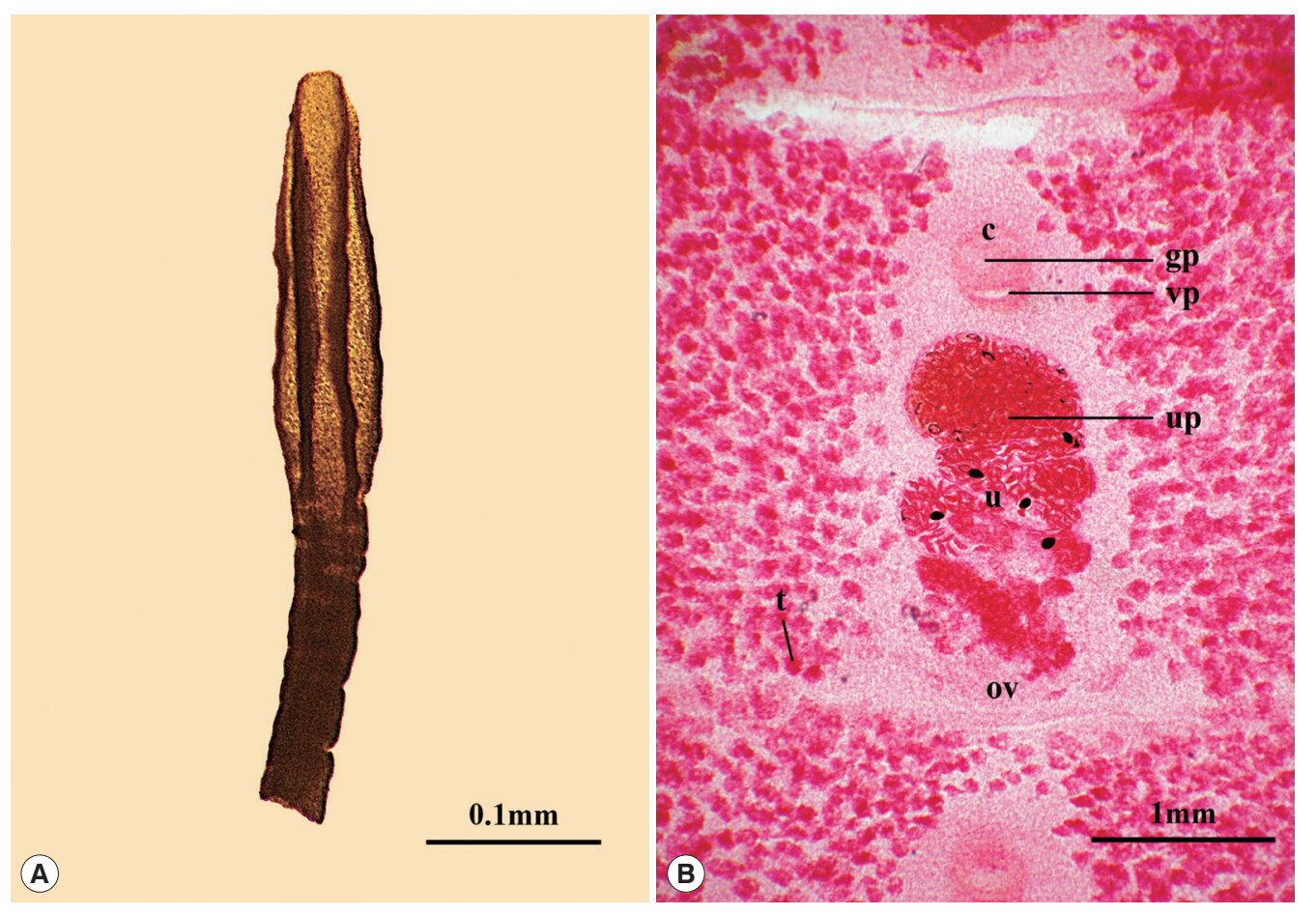

Fig. 1. Spirometra decipiens collected from a road killed stray cat in Chungbuk, Korea. (A) Scolex. (B) Whole-mounted specimen of proglottids showing the uterus and cirrus pouch (×40). Abbreviations; cirrus (c), uterus (u), genital pore (gp), vaginal pore (vp), uterine pore (up), ovaries (ov) and testes (t) (carmine stain). 
Mature proglottids measured $4.2 \pm 0.03 \mathrm{~mm}$ in width and $1.70 \pm 0.01 \mathrm{~mm}$ in length (Fig. 1B). The uterus was coiled 3-4 times, the end of the uterus was ball-shaped, and the vaginal aperture shaped as a crescent moon was closer to the cirrus aperture than to the uterus aperture (Fig. 1B). The uterine ball measured $457.7 \pm 4.8 \mu \mathrm{m}$ in diameter, and uterine pores were on the midline behind the anterior margin of the uterine ball. The cirrus pouch measured $260.3 \pm 2.6 \mu \mathrm{m}$ in diameter. The genital pore was situated ventrally on the midline in the anterior $1 / 5$ segment. The testes measured $71.4 \pm 0.7 \mu \mathrm{m}$ maximum diameter (Fig. 1B) (Table 1).

PCR amplification and direct sequencing of the cox 1 target fragment (377 bp in length and corresponding to positions 769-1,146 bp of the cox1 gene) were performed using total genomic DNA extracted from 134 specimens. The partial cox1 sequences (377 bp) of the scolices $(n=134)$ and proglottids ( $\mathrm{n}=41$ ) showed $99.0 \%$ similarity to the $S$. decipiens reference sequence (GenBank no. KJ599679) and $89.3 \%$ similarity to the $S$. erinaceieuropaei reference sequence (GenBank no. KJ599680). The difference in nucleotide sequence of the partial cox1 gene between the specimens and $S$. decipiens was $0.1 \%$,

Table 1. Morphological features of spirometrid tapeworms recovered from a road killed cat

\begin{tabular}{lc}
\hline Organs & Size \\
\hline Scolex $(\mathrm{n}=116)$ & $2.74 \pm 0.43 \mathrm{~mm}$ (diameter) \\
& $0.73 \pm 0.11 \mathrm{~mm}$ (length) \\
Mature proglottids $(\mathrm{n}=41)$ & $4.19 \pm 0.02 \mathrm{~mm}$ (width) \\
& $1.70 \pm 0.01 \mathrm{~mm}$ (length) \\
Uterus & $3-4$ loops \\
Uterine ball & $457.6 \pm 4.77 \mu \mathrm{m}$ (diameter) \\
Cirrus pouch & $260.3 \pm 2.62 \mu \mathrm{m}$ (diameter) \\
Genital pore & $115.7 \pm 1.55 \mu \mathrm{m}$ (diameter) \\
Testes & $71.4 \pm 0.71 \mu \mathrm{m}$ (diameter) \\
\hline
\end{tabular}

while that with S. erinaceieuropaei was $9.7 \%$. The pairwise genetic distance between the specimens (scolices and proglottids) and $S$. decipiens was 0.0026 , while that with S. erinaceieuropaei was $0.0079 \pm 0.0237$ (Table 2 ).

In the present study, all scolices and proglottids were identified as $S$. decipiens using nucleotide sequence analysis of the mitochondrial cox1 gene. Pairwise genetic distances between $S$. decipiens and S. erinaceieuropaei isolated from the cat examined were calculated as being the same as those of previous studies of snakes $(0.0079 \pm 0.0237)$ [8]. The major distinguishing morphologic characteristics of Spirometra species are the number of uterine coils. The mature proglottids in this study were identical with those of $S$. decipiens by the morphological characteristics of reproductive organs, especially 3-4 spiral rotations of the uterus.

The taxonomy of the genus Spirometra has been controversial because of its species classification. Approximately 38 species of the genus Spirometra have been reported from intestinal parasites of canines and felines [9,11-13]. All species in the genus Spirometra were considered synonymous with S. erinaceieuropaei found in Asian countries, including China, Japan, and Korea [11-13]. The mitochondrial DNA sequences of the genus Spirometra have been used as identification and genetic variation of species to resolve uncertain taxonomic status. The nucleotide sequence variation of $S$. erinaceieuropaei was reported by Okamoto et al. (<2.6\%) [15] and Liu et al. (0.0-3.1\%) [16]. The complete mitochondrial genomes of genus Spirometra have been recently published for $S$. erinaceieuropaei and $S$. decipiens based on morphological analyses of Spirometra tapeworms by Eom et al. [7]. Thirty-seven human sparganosis were reported from Africa, including 34 from South Sudan and 3 from Ethiopia by Eberhard et al. [17]. The partial cox1 nucleotide divergence of Asian isolate identified as $S$. decipiens dif-

Table 2. Pairwise genetic distance of the mitochondrial cox1 gene of Spirometra specimens isolated from a cat in comparison with Spirometra spp., Diphyllobothrium nihonkaiense, and Diphyllobothrium latum

\begin{tabular}{|c|c|c|c|c|c|c|}
\hline Species & Scolices & Proglottids & $\begin{array}{l}\text { Spirometra } \\
\text { decipiens }\end{array}$ & $\begin{array}{c}\text { Spirometra } \\
\text { erinaceieuropaei }\end{array}$ & $\begin{array}{l}\text { Diphyllobothrium } \\
\text { nihonkaiense }\end{array}$ & $\begin{array}{c}\text { Diphyllobothrium } \\
\text { latum }\end{array}$ \\
\hline \multicolumn{7}{|l|}{ Scoleces $(n=134)$} \\
\hline Proglottids $(n=41)$ & $0.00265 \pm 0.00000$ & & & & & \\
\hline Spirometra decipiens & $0.00265 \pm 0.00000$ & $0.00265 \pm 0.00000$ & & & & \\
\hline $\begin{array}{l}\text { Spirometra } \\
\text { erinaceieuropaei }\end{array}$ & $0.00796 \pm 0.02369$ & $0.00796 \pm 0.02369$ & $0.01031 \pm 0.02251$ & & & \\
\hline $\begin{array}{l}\text { Diphyllobothrium } \\
\text { nihonkaiense }\end{array}$ & $0.15467 \pm 0.00218$ & $0.15467 \pm 0.00218$ & $0.15201 \pm 0.00218$ & $0.14834 \pm 0.00284$ & & \\
\hline Diphyllobothrium latum & $0.16490 \pm 0.00099$ & $0.16490 \pm 0.00099$ & $0.16490 \pm 0.00099$ & $0.15429 \pm 0.00099$ & $0.07206 \pm 0.00099$ & \\
\hline
\end{tabular}

Genetic distance $($ mean \pm SD) calculated by the neighbor-joining method using Kimura's 2-parameter model based on 377 bp partial cox1 sequences. 
fered from South America isolate (17.8-19.2\%), but it was closely related to the Asian isolates of S. erinaceieuropaei (0.34.0\%) [18]. The South American isolates of Spirometra species were revealed as neither $S$. erinaceieuropaei nor $S$. decipiens by mitochondrial cox1 sequence analyzed. Those cases differed from Asian isolates of S. erinaceieuropaei and S. decipiens as well as those of African countries by analysis of mitochondrial DNA sequence data. Above studies provoked epidemiological questions concerning what is the geographical distribution of Spirometra spp., including S. erinaceieuropaei and S. decipiens? In addition, why is a high level of nucleotide variation found among Brazilian, Asian, and African isolates? The taxonomic status of Spirometra species has been unsettled. Further studies are needed investigations using a number of global samples to clarify the taxonomy of the genus Spirometra.

The final hosts of Spirometra spp. are known to be carnivorous animals such as cats and dogs; however, we only found $S$. decipiens in the absence of other Spirometra species, including $S$. erinaceieuropaei in natural infections in cats in those studies. A previous study identified $S$. decipiens plerocercoids in terrestrial snakes from Korea and China, without any exceptions [9]. In another report concerning human sparganosis cases, 35 and 15 instances of S. erinaceieuropaei and S. decipiens, respectively, were identified, revealing approximately twice as many cases of S. erinaceieuropaei compared to S. decipiens [6]. These findings indicated not only the presence of epidemiological discrepancies between the prevalence of Spirometra spp. in humans and intermediates or final hosts, but also that the infection route of $S$. erinaceieuropaei for humans remained unclear.

Since it is very difficult to identify the species of eggs of Spirometra using morphology alone, DNA-based analysis is considered necessary to taxonomically classify the genus Spirometra. Many studies had documented Spirometra spp. in surveys undertaken among cats before $S$. decipiens had been reported in Korea [1-5]. Those previous studies may need to be reexamined using molecular techniques in an effort to better understand the epidemiological status of Spirometra spp. in Korea. Although many studies have examined the prevalence of intestinal parasites in stray cats, none of the recent reports have indicated a heavy infection of Spirometra species in Korea as detailed in our present study. Moreover, all worms were identified as $S$. decipiens rather than $S$. erinaceieuropaei by morphological and molecular analyses.

In conclusion, we report a stray cat heavily infected with $S$. decipiens identified by mitochondrial cox1 sequence analysis and morphological examinations of the adult worms. These findings suggested that stray cats play an important role of a reservoir host for $S$. decipiens inducing human sparganosis in Korea.

\section{ACKNOWLEDGMENT}

This work was supported by the National Research Foundation of Korea (no. 2014R1A1A2004933). And the materials used were provided by the parasite resource bank of Korea.

\section{CONFLICT OF INTEREST}

We have no conflict of interest related to this work.

\section{REFERENCES}

1. Kang HJ. Studies on the parasitic helminths of the cats in western province of Kyung Sang Nam-do. Res Bull Chinju Agric Coll 1967; 6: 91-96 (in Korean).

2. Huh S, Sohn WM, Chai JY. Intestinal parasites of cats purchased in Seoul. Korean J Parasitol 1993; 31: 371-373.

3. Yang HJ, Park TW, Cheon SJ, Yoon YB, Kim NJ, Park BK, Kim CS. Internal parasites of cats in Iri and its vicinity. Korean J Vet Serv 1995; 18: 33-40 (in Korean).

4. Sohn WM, Chai JY. Infection status with helminthes in feral cats purchased from a market in Busan, Republic of Korea. Korean J Parasitol 2005; 43: 93-100.

5. Chai JY, Bahk YY, Sohn WM. Trematodes recovered in the small intestine of stray cats in the Republic of Korea. Korean J Parasitol 2013; 51: 99-106.

6. Jeon HK, Park HS, Lee DM, Choe SJ, Kim KH, Huh S, Sohn WM, Chai JY, Eom KS. Human infections with Spirometra decipiens plerocercoids identified by morphologic and genetic analyses in Korea. Korean J Parasitol 2015; 53: 299-305.

7. Eom KS, Park HS, Lee DM, Choe SJ, Kim KH, Jeon HK. Mitochondrial genome sequences of Spirometra erinaceieuropaei and $S$. decipiens (Cestoidea: Diphyllobothriidae). Korean J Parasitol 2015; 53: 455-463.

8. Jeon HK, Park HS, Lee DM, Choe SJ, Kim KH, Sohn WM, Eom KS. Genetic identification of Spirometra decipiens plerocercoids in terrestrial snakes from Korea and China. Korean J Parasitol 2016; 54: 181-185.

9. Faust EC, Campbell HE, Kellogg CR. Morphological and biological studies on the species of Diphyllobothrium in China. Am J Hyg 1929; 9: 560-583.

10. Jeon HK, Park HS, Lee DM, Choe SJ, Sohn WM, Eom KS. Molecular detection of Spirometra decipiens in the United States. Korean J Parasitol 2016; 54: 503-507.

11. Wardle RA, McLeod JA. The Zoology of Tapeworms. Minneapo- 
lis, USA. Minnesota University Press. 1952, pp 559-615.

12. Yamaguti S. Systema Helminthum. Vol. II. The cestodes of vertebrates. New York, USA. Interscience Publishers. 1959, p 358361.

13. Kamo H. Guide to Identification of Diphyllobothriid Cestodes. Tokyo, Japan. Gendai Kikaku. 1999, p 1-146 (in Japanese).

14. Iwata S. Experimental and morphological studies of Manson's tapeworm, Diphyllobothrium erinacei Rudolphi. Special reference with its scientific name and relationship with Sparganum proliferum Ijima. Progr Med Parasitol Jpn 1972; 4: 536-590.

15. Okamoto M, Iseto C, Shibahara T, Sato MO, Wandra T, Craig PS, Ito A. Intraspecific variation of Spirometra erinaceieuropaei and phylogenetic relationship between Spirometra and Diphyllobothrium inferred from mitochondrial CO1 gene sequence. Parasitol
Int 2007; 56: 235-238.

16. Liu W, Zhao GH, Tan MY, Zeng DL, Wang KZ, Yuan ZG, Lin RQ, Zhu XQ, Liu Y. Survey of Spirometra erinaceieuropaei spargana infection in the frog Rana nigromaculata of the Hunan province of China. Vet Parasitol 2010; 173: 152-156.

17. Eberhard ML, Thiele EA, Yembo GE, Yibi MS, Cama VA, RuizTiben E. Thirty-seven human cases of sparganosis from Ethiopia and South Sudan caused by Spirometra spp. Am J Trop Med Hyg 2015; 93: 350-355.

18. Almeida GG, Coscarelli D, Melo MN, Melo AL, Pinto HA. Molecular identification of Spirometra spp. (Cestoda: Diphyllobothiidae) in some wild animals from Brazil. Parasitol Int 2016; 65: 428-431. 
